\title{
On the Nonlinear Nature of the Turbulent $\alpha$-Effect
}

\author{
Fausto Cattaneo \\ Department of Astronomy and Astrophysics, University of Chicago, \\ 5640 S. Ellis Ave., Chicago, IL 60637, U.S.A.
}

David W. Hughes

Department of Applied Mathematics, University of Leeds, Leeds LS2 9JT, U.K.

Jean-Claude Thelen

Department of Astronomy and Astrophysics, University of Chicago, 5640 S. Ellis Ave., Chicago, IL 60637, U.S.A.

Abstract. Galactic magnetic fields are, typically, modelled by meanfield dynamos involving the $\alpha$-effect. Here we consider, very briefly, some of the issues involving the nonlinear dependence of $\alpha$ on the mean field.

\section{Introduction}

Large-scale galactic magnetic fields are usually ascribed to be the result of some sort of hydromagnetic dynamo action (see, for example, the monograph by Ruzmaikin, Shukurov \& Sokoloff 1988 and the review by Beck et al. 1996). Galactic dynamos have, almost without exception, been modelled by $\alpha-\omega$ mean field dynamos: in such a prescription, poloidal field is sheared by the differential rotation, $\omega$, to produce a toroidal field, and, conversely, the reverse (toroidal to poloidal) step is achieved by the so-called ' $\alpha$-effect' of mean field electrodynamics. A non-zero $\alpha$-effect is attributable to a lack of reflexional symmetry in the underlying turbulence; in the galactic context such turbulence may be the consequence of exploding supernovae influenced by the galactic rotation.

Standard mean-field formalism is based essentially on a kinematic treatment in which the magnetic field has no influence on the velocity field. In reality, of course, dynamo-generated fields will eventually attain sufficient strength to react back on the driving flow. Consequently, one of the important points to address is the dependence of the $\alpha$-effect on the mean (large-scale) magnetic field $B_{0}$ and also, crucially, its dependence on the magnetic Reynolds number $R m$. This is a controversial issue which, in its simplest form, can be reduced to asking whether $\alpha$ satisfies a relation of the form

$$
\alpha=\frac{\alpha_{0}}{1+B_{0}^{2} /\left\langle u^{2}\right\rangle}
$$


where $\alpha_{0}$ is the turbulent value and the magnetic field strength $B_{0}$ is measured in units of the Alfvén speed, or a relation of the form

$$
\alpha=\frac{\alpha_{0}}{1+R m^{\gamma} B_{0}^{2} /\left\langle u^{2}\right\rangle},
$$

for some $O(1)$ value of $\gamma$.

Given that $R m$ in a galactic context is very large (estimates for $R m$ based purely on collisional processes are $O\left(10^{14}\right)$, those based on ambipolar diffusion are $O\left(10^{6}\right)$ ), expressions (1) and (2) lead to very different conclusions; the former implies that the large-scale field can reach equipartition strength before $\alpha$ is suppressed, the latter that suppression occurs for a large-scale field smaller than equipartition by a factor of $\mathrm{Rm}^{\gamma / 2}$.

\section{Physical Considerations}

\subsection{Two-dimensional Turbulent Diffusion}

To illustrate some of the crucial ideas it is instructive to consider the simpler, but related, problem of the diffusion of a planar magnetic field due to a twodimensional turbulent flow. Here dynamo action is impossible and decay of the field inevitable. The turbulent diffusion time $t_{T}$ for a kinematic magnetic field over a scale $L$ is given by the classical result

$$
t_{T} \approx L^{2} / U \ell
$$

where $\ell$ is the scale of the energy-containing eddies. The dynamic decay time (i.e. taking into account the Lorentz force) can however be significantly enhanced (Vainshtein \& Cattaneo 1992). If $B_{0}$ is the large-scale component of the field then, as shown by Zeldovich (1957), $\left\langle|\mathbf{B}|^{2}\right\rangle \approx R m B_{0}^{2}$; i.e. the fluctuating field is significantly $\left(O\left(R \mathrm{~m}^{1 / 2}\right)\right)$ stronger than the large-scale field. Turbulent diffusion occurs through the generation of small-scale fields that eventually are annihilated by molecular processes. However, the generation of small-scale fields is inextricably linked with the generation of strong fields. If the fields so generated attain equipartition with the energy-containing eddies of the flow at scales larger than the diffusive scale then severe inhibition of the diffusive process occurs. Indeed, the dynamic timescale, $t_{D}$, for the decay of the magnetic field satisfies

$$
t_{D} \approx \frac{L^{2}}{\eta}\left(\frac{1}{R m}+\frac{1}{M^{2}+1}\right)
$$

(Cattaneo \& Vainshtein 1991), where $M$ is the Alfvénic Mach number of the large-scale field $(M>1(<1)$ implying that the large-scale field is less than (greater than) equipartition strength). The most significant feature of equation (4) is that, in accordance with the issues discussed above, only very weak largescale fields $\left(M \lesssim R m^{1 / 2}\right)$ are needed to influence strongly the diffusive process.

Cattaneo (1994) has reconsidered this problem to investigate the physical mechanism behind the suppression of diffusion. Taylor (1921) showed that for a purely passive scalar contaminant, the turbulent diffusivity $D$ could be expressed 
in terms of the Lagrangian displacement of fluid particles $\xi$ as

$$
D=\frac{1}{4} \frac{d}{d t}\left\langle\xi^{2}\right\rangle
$$

Cattaneo (1994) showed that the role of a (weak) large-scale magnetic field is to suppress the tendency of particles to undergo a random walk and hence to suppress the turbulent diffusivity of the field.

\subsection{Three-dimensional Flows}

For three-dimensional flows the situation is more complicated with the possibility (forbidden in 2D) of dynamo action. As shown by Moffatt (1974), at infinite $R m, \alpha$ may be obtained formally from the Cauchy solution as $\alpha=-d\langle\xi \cdot \nabla \times$ $\xi\rangle / d t$, showing clearly that $\alpha$, like the turbulent diffusivity, is also a transport coefficient. It is then certainly conceivable that the generation of strong magnetic fields on small scales could inhibit $\alpha$ in an analogous manner to the suppression of turbulent diffusion discussed above. To determine whether this is indeed the case requires a combination of rigorous analysis and careful numerical simulations.

\section{Numerical Results and Discussion}

Cattaneo, Hughes \& Thelen (2001) have considered the dependence of $\alpha$ on both $B_{0}$ and $R m$, for the flow investigated by Cattaneo \& Hughes (1996). Their results strongly support a nonlinear $\alpha$-effect of the form of equation (2). Despite these compelling results, the subject, nonetheless, remains controversial and a number of papers have appeared arguing instead for expression (1) (e.g. Field, Blackman \& Chou 1999). A full discussion of the issues involved, some of which are quite complex and beyond the scope of a brief communication such as this, will appear in our forthcoming papers.

\section{References}

Beck, R., Brandenburg, A., Moss, D., Shukurov, A. \& Sokoloff, D. 1996, ARA\&A, 34,155

Cattaneo, F. 1994, ApJ, 434, 200

Cattaneo, F. \& Hughes, D.W. 1996, Phys. Rev. E, 54, R4532

Cattaneo, F. \& Vainshtein, S.I. 1991, ApJ, 376, L21

Cattaneo, F., Hughes, D. W. \& Thelen, J.-C. 2001, J. Fluid Mech. (submitted)

Field, G. B., Blackman, E. G. \& Chou, H. 1999, ApJ, 513, 638

Moffatt, H. K. 1974, J. Fluid Mech., 65, 1

Ruzmaikin, A. A., Shukurov, A. M. \& Sokoloff, D.D. 1988, Magnetic Fields of Galaxies (Kluwer: Dordrecht)

Taylor, G.I. 1921, Proc. London Math. Soc. A20, 196

Vainshtein, S. I. \& Cattaneo, F. 1992, ApJ, 393, 165

Zeldovich, Ya. B. 1957, Soviet Phys.-JETP, 4, 460 